\title{
The role of number of meals, coffee intake, salt and type of cookware on colorectal cancer development in the context of the Mediterranean diet
}

\author{
Niki Kontou ${ }^{1,2}$, Theodora Psaltopoulou ${ }^{3}$, Nick Soupos ${ }^{3}$, Evangelos Polychronopoulos ${ }^{1}$, \\ Athena Linos ${ }^{3}$, Dimitrios Xinopoulos ${ }^{2}$ and Demosthenes B Panagiotakos ${ }^{1, *}$ \\ 'Department of Nutrition and Dietetics, Harokopio University, 70 Eleftheriou Venizelou Street, \\ 17671 Athens, Greece: ${ }^{2}$ Saint Savvas Cancer Hospital, Athens, Greece: ${ }^{3}$ School of Medicine, \\ University of Athens, Athens, Greece
}

Submitted 7 January 2012: Final revision received 29 May 2012: Accepted 4 June 2012: First published online 8 August 2012

\begin{abstract}
Objective: To investigate the association between dietary behaviours and colorectal cancer (CRC) in the context of the Mediterranean diet.

Design: Case-control study.

Setting: All patients (cases) were recruited from Saint Savvas Cancer Hospital and Alexandra General Hospital in Athens, Greece. Controls were voluntarily selected from the general population and matched to cases by age group ( \pm 10 years) and sex.

Subjects: Two hundred and fifty cases with newly diagnosed CRC (mean age 63 (SD 12) years, 59.6\% males) and 250 controls matched on age and sex were studied. A standardized questionnaire assessing sociodemographic, clinical, lifestyle, dietary characteristics and nutritional behaviours was applied. Multiple logistic regression analysis was used to evaluate the aforementioned factors in addition to the MedDietScore (an index that evaluates adherence to the Mediterranean diet) on CRC development.

Results: The higher the daily number of meals, the lower the likelihood of having CRC (OR $=0 \cdot 74,95 \%$ CI 0.61, 0.89); coffee drinking was associated with higher likelihood of having CRC (OR $=3 \cdot 27,95 \%$ CI $1 \cdot 09,9 \cdot 8)$; the use of non-stick cookware was positively associated with $\mathrm{CRC}(\mathrm{OR}=1 \cdot 57,95 \%$ CI $1 \cdot 02,2 \cdot 4)$. However, these associations slightly lost their significance when adherence to the Mediterranean diet was taken into account. Moreover, a $1 / 75$ increase in the modified-MedDietScore plus the aforementioned nutritional behaviours was associated with $13 \%$ lower odds (95\% CI 0.83, 0.91, $P<0 \cdot 001$ ) of having CRC. Conclusions: Nutritional behaviours in addition to dietary habits should be taken into account in detecting individuals prone to the development of CRC.
\end{abstract}

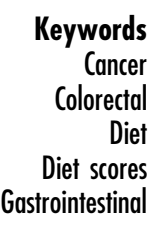

According to GLOBOCAN data from the International Agency for Research on Cancer on incidence of major cancer types, colorectal cancer (CRC) ranked the third highest worldwide in 2008. Based on the same report, CRC incidence was in first place in Europe and in third place in Greece $^{(1)}$. In the last two decades nutritional epidemiology has turned its interest from the study of nutrients and foods to the holistic approach of relating diet to chronic diseases, based on the fact that people consume a variety of foods with complex combinations of micro- and macronutrients (i.e. dietary patterns) and not single nutrients ${ }^{(2)}$. A healthy overall dietary pattern approach is now considered to be the cornerstone for the prevention of CVD, cardiometabolic disorders ${ }^{(2,3)}$ and some types of cancer, such as of the breast, colorectum and upper aerodigestive tract ${ }^{(4-10)}$. The most well studied and documented dietary pattern, mainly concerning CVD, is the Mediterranean diet. However, some dietary behaviours, like drinking coffee, adding salt at the table, salted foods, meal frequency and cooking procedures, have not been included in dietary patterns, although they have been associated with the development of chronic diseases, like cancer. Specifically, epidemiological studies have shown that high intake of calcium and vitamin $\mathrm{D}^{(11,12)}$ seems to have a protective effect on CRC risk and coffee intake $^{(13-15)}$ a conflicting effect, while frequent meals ${ }^{(16,17)}$ and systematic physical activity ${ }^{(18,19)}$ probably have modest protective effect. Added table salt or salted foods have been highly correlated with gastric cancer, but not with CRC. Despite this finding, a low-sodium diet is considered 
to be beneficial for human health ${ }^{(20)}$. Furthermore, nonstick cookware, and specifically the coating, has been related to several types of tumour in animal studies, mainly due to the release of perfluorooctanoic acid (PFOA) and perfluorooctanesulfonate $(\mathrm{PFOS})^{(21)}$. There is also a concern due to the fact that non-stick cookware is mostly used for cooking at high temperatures, which is in turn related to increased production of heterocyclic aromatic amines (HCA) and polycyclic aromatic hydrocarbons (PAH). HCA and PAH have been found to interact with the polymorphisms in carcinogen-metabolizing enzymes ${ }^{(22)}$. Thus, although a healthy dietary pattern may be associated with cancer prevention, it remains unclear whether the diet as a whole, the specific components of the pattern or other nutritional behaviours are responsible for this effect ${ }^{(10)}$. Taking also into account that computer simulation models have underlined the importance of lifestyle modification finding that it could have a comparable, or perhaps even greater, up to $50 \%$, impact on CRC mortality rates compared with increased chemotherapy use during the next $10-20$ years ${ }^{(23,24)}$ - the role of dietary habits and behaviours in cancer development is potentially substantial.

Thus, the aim of the present work was to evaluate whether certain dietary behaviours, i.e. number of meals consumed daily, use of table salt, consumption of meat products highly preserved in salt (like ham, bacon, sausages), coffee and tea drinking, times of fast food consumption per week, intake of vitamin or other dietary supplements and use of non-stick cookware, are associated with CRC development in the context of a healthy dietary pattern, the Mediterranean diet.

\section{Methodology}

\section{Study design and sample}

This is a case-control study that was conducted from December 2009 to December 2010. The study enrolled 500 participants (mean age 63 (SD 12) years, 59\% males); of them, 250 were patients with a first diagnosis of CRC who consecutively visited Saint Savva Cancer Hospital and Alexandra General Hospital in Athens (cases) and 250 were individuals without any clinical symptoms or signs of any type of cancer in their medical history as evaluated by the study's investigators (controls). Cases were selected from the admission list of the aforementioned hospitals and the diagnosis of first developed CRC was defined by biopsy after surgical resection. Controls were voluntarily selected from the general population (i.e. workplaces or home) and were matched to the cases by age group ( \pm 10 years), sex and area of residence. Data for both cases and controls were collected through face-to-face interviews, by the investigators of the study. The participation rate of those enrolled was high, reaching 95\% for cases and $80 \%$ for controls.

The number of individuals enrolled ( $n$ 500) was decided through power analysis, in order to evaluate (two sided) odds ratios equal to 0.75 with statistical power greater than $77 \%$ at significance level less than $5 \%$. The investigators of the study were all trained and performed the evaluation of clinical symptoms and signs in the series of cases and controls.

\section{Bioetbics}

Before the face-to-face interview, all participants were informed about the aims of the study, agreed to give the requested information and signed an informed consent. The study's protocol was approved by the hospitals' ethics committees (no. 15299/416/22-12-2009).

\section{Assessment of certain nutritional behaviours and adberence to the Mediterranean diet}

Participants completed a standardized questionnaire that was developed for the purposes of the study and included demographic, clinical, lifestyle, dietary and nutritional behaviour questions. The dietary questionnaire used was based on a previously developed validated and repeatable FFQ. In brief, the FFQ included sixty-nine questions regarding the frequency of consumption of all main food groups and beverages usually consumed and seven questions regarding eating behaviours ${ }^{(25)}$. Among others, the questionnaire included questions about the number of meals regularly consumed each day (measured on a quantitative scale; i.e. $1,2,3,4,5,>5$ ), use of table salt during daily meals (yes, no), frequency of consumption of meat products highly preserved in salt (like ham, bacon, sausages), coffee and tea drinking (i.e. in cups of $150 \mathrm{ml} / \mathrm{d}$ and type of coffee or tea consumed), times of fast food consumption per week, intake of vitamin or other dietary supplements, as well as use of non-stick cookware (i.e. never, sometimes, always). For the analysis, all reported types of coffee (instant, brewed coffee, Greek type, cappuccino or filtered, as well decaffeinated) were adjusted for 1 cup $=150 \mathrm{ml}$ and caffeine concentration of $28 \mathrm{mg} / 150 \mathrm{ml}$ (with the exception of decaffeinated).

Adherence to the Mediterranean diet was evaluated by the MedDietScore ${ }^{(26)}$. In brief, the MedDietScore is an eleven-item dietary score that includes questions about the weekly or monthly consumption of the following nine food groups that characterize the traditional Mediterranean dietary pattern: (i) non-refined cereals (wholegrain bread and pasta, brown rice, etc.), (ii) potatoes, (iii) fruit, (vi) vegetables, (v) legumes, (vi) fish, (vii) meat and meat products, (viii) poultry, (ix) full-fat dairy products (such as cheese, yoghurt, milk); as well as: (x) olive oil use in daily cooking and (xi) alcohol drinking. For scoring the frequency of consumption of the aforementioned food groups the methodology proposed by Kourlaba and Panagiotakos was applied $^{(27)}$; in particular, for the food groups presumed to be close to the Mediterranean dietary pattern, scores from 0 to 5 were given for never to frequent intake, whereas for food groups that were considered to be away from the Mediterranean diet, the reverse scoring (i.e. from 5 to 0 ) 
was assigned. Based on the fact that a J-shaped association of alcohol drinking with cardiovascular risk exists, usual alcohol intake (i.e. red or white wine, beer, whisky or any other kind of liquor, i.e. typical Greek beverages like ouzo and tsipouro, in a glass equal to $100 \mathrm{ml}$, with an equivalent of $12 \mathrm{~g}$ of ethanol) was evaluated with a nonmonotonic scoring system (i.e. assigning a score of 5 for consumption of $<300 \mathrm{ml} / \mathrm{d}$, a score of 0 for no consumption or for consumption of $>700 \mathrm{ml} / \mathrm{d}$, and a score of 4 to 1 for consumption of 600-700, 500-600, 400-500 and $300-400 \mathrm{ml} / \mathrm{d}$, respectively). Specifically, for alcohol intake, data on consumption of alcohol (defined as daily alcohol consumption frequency) and the usual type of alcohol consumed were recorded for the last year prior to diagnosis. Thus, the theoretical range of the MedDietScore was $0-55$ and higher values indicate greater adherence to the Mediterranean diet. Recently, the MedDietScore has been applied in patients with gastrointestinal tract cancers and showed good accuracy ${ }^{(28)}$.

Furthermore, a diet and nutrition behaviour score (for simplicity, called the modified-MedDietScore) was developed that included the MedDietScore and the nutritional habits that showed statistical significance with the investigated outcome (i.e. number of daily meals, use of table salt, coffee drinking and use of non-stick cookware), as well. Specifically, concerning the nutritional behaviour components included in the modified version of diet score, daily coffee consumption was a variable where a non-monotonic function was applied assigning a score of 0 for no daily intake and scores of 5 to 2 for $2-3,1,4-5$ and $>5$ cups/d. The daily number of meals followed a monotonic scoring system, giving a score of 0 for $1 \mathrm{meal} / \mathrm{d}$ and a score of 5 for $>5$ meals $/ \mathrm{d}$. Use of non-stick cookware and added table salt were scored using the reverse scale due to the fact that they seem to be related to cancer epidemiology, assigning the score of 5 when someone reported no use of non-stick cookware (no added table salt) and 0 for daily use of non-stick cookware (daily addition of table salt). Thus, the modified-MedDietScore theoretically ranged from 0 to 75 ; higher values of this score suggest greater adherence to the Mediterranean diet and healthier nutritional behaviours.

\section{Otber measurements}

Age and sex of the participants were recorded. Weight and height were measured using standard procedures and BMI $\left(\mathrm{kg} / \mathrm{m}^{2}\right)$ was calculated as weight (in kilograms) divided by the square of height (in metres). Based on the fact that mean age-standardized BMI has increased globally since 1980 to 2008 , representing a $0 \cdot 4-0.5 \mathrm{~kg} / \mathrm{m}^{2}$ increase per decade in men and women ${ }^{(29)}$, a threshold of $27 \mathrm{~kg} / \mathrm{m}^{2}$ was used to define excess body mass. Physical activity status (sedentary; low active, expended energy $<16.7 \mathrm{~kJ}$ $(<4 \mathrm{kcal}) / \mathrm{min}$, i.e. walking slowly, cycling stationary, light stretching, etc.; moderately active, expended energy
$16 \cdot 7-29 \cdot 3 \mathrm{~kJ}$ (4-7 kcal)/min, i.e. walking briskly, cycling outdoors, swimming moderate effort, etc.; highly active, expended energy $>29 \cdot 3 \mathrm{~kJ}(7 \mathrm{kcal}) / \mathrm{min}$, i.e. walking briskly uphill, long-distance running, cycling fast or racing, swimming fast crawl, etc.) and smoking habits (i.e. current, former, ever or never, and cigarettes smoked per day) were also recorded. History of hypertension, diabetes, dyslipidaemia and family history of CRC were also recorded for both cases and controls. Metabolic syndrome was defined following the criteria of the National Cholesterol Education Program Adult Treatment Panel III ${ }^{(30)}$.

\section{Statistical analysis}

Continuous variables according to the clinical status of the participants are presented as means and standard deviations. Comparisons of components of the MedDietScore between cases and controls were performed using the independent-samples $t$ test or the Mann-Whitney test depending of the normality or not of the tested variable. Multiple logistic regression analysis was applied to evaluate the studied nutritional behaviours as well as their additive role on the MedDietScore in relation to the presence of $\mathrm{CRC}$; results are presented as odds ratios and their 95\% corresponding confidence intervals. The C-statistic, a measure of how well a prediction rule (i.e. the diet score) can correctly discriminate patients from controls, was also calculated (values close to 1 suggest good correct classification rate of the model). All reported $P$ values were based on two-sided tests and compared with a significance level of $0 \cdot 05$. Statistical calculations were performed using the SPSS 18 statistical software package (SPSS Inc.).

\section{Results}

\section{Dietary and nutritional bebaviours in relation to colorectal cancer}

As can be seen in Table 1, the number of meals consumed daily, tea drinking and the use of table salt after cooking differed significantly between cases and controls; whereas coffee drinking, consumption of meat products highly preserved in salt, times of fast food consumption per week, use of non-stick cookware and intake of vitamin or other dietary supplements did not differ between cases and controls. It should be mentioned that although tea intake differed between the two groups, overall tea intake was pretty low (less than 20\%) to be considered in further analyses.

However, residual confounding may exist. Thus, multiple logistic regression analysis after adjusting for age, sex, BMI, physical activity status, smoking habits and family history of $\mathrm{CRC}$ revealed that: the higher the number of meals daily, the lower the likelihood of having CRC; drinking 4-5 cups of coffee/d was associated with a higher likelihood of having $\mathrm{CRC}$ as compared with no drinking; the use of non-stick cookware was positively associated with CRC; and added 
Table 1 Dietary habits and nutritional behaviours in a sample of colorectal cancer (CRC) cases ( $n$ 250) and controls group-matched for age and sex ( $n$ 250), Athens, Greece, December 2009-December 2010

\begin{tabular}{|c|c|c|c|c|c|}
\hline & \multicolumn{2}{|c|}{ CRC cases } & \multicolumn{2}{|c|}{ Controls } & \multirow[b]{2}{*}{$P$} \\
\hline & Mean & SD & Mean & SD & \\
\hline MedDietScore (0-55) & $29 \cdot 4$ & 4 & $31 \cdot 1$ & 4 & $<0.001$ \\
\hline \multicolumn{6}{|l|}{ Nutrition behaviour variables } \\
\hline Number of meals per day & 3 & $1 \cdot 0$ & 4 & $1 \cdot 1$ & $<0.001$ \\
\hline Coffee drinking (\%) & & & & & 0.25 \\
\hline None & \multicolumn{2}{|c|}{$12 \cdot 9$} & \multicolumn{2}{|c|}{$15 \cdot 6$} & \\
\hline $1 \mathrm{cup} / \mathrm{d}$ & \multicolumn{2}{|c|}{$32 \cdot 9$} & \multicolumn{2}{|c|}{$35 \cdot 2$} & \\
\hline $2-3$ cups/d & \multicolumn{2}{|c|}{$44 \cdot 6$} & \multicolumn{2}{|c|}{$44 \cdot 0$} & \\
\hline $4-5$ cups/d & \multicolumn{2}{|c|}{$7 \cdot 6$} & \multicolumn{2}{|c|}{$3 \cdot 2$} & \\
\hline $5+$ cups $/ d$ & \multicolumn{2}{|c|}{$2 \cdot 0$} & \multicolumn{2}{|c|}{$2 \cdot 0$} & \\
\hline Tea drinking (\%) & \multirow{2}{*}{\multicolumn{2}{|c|}{$75 \cdot 7$}} & \multirow{2}{*}{\multicolumn{2}{|c|}{$73 \cdot 0$}} & 0.04 \\
\hline None & & & & & \\
\hline $1 \mathrm{cup} / \mathrm{d}$ & \multicolumn{2}{|c|}{$22 \cdot 3$} & \multicolumn{2}{|c|}{$20 \cdot 2$} & \\
\hline $2-3$ cups/d & \multicolumn{2}{|c|}{$1 \cdot 6$} & \multicolumn{2}{|c|}{$6 \cdot 5$} & \\
\hline 4-5 cups/d & \multicolumn{2}{|c|}{0.4} & \multicolumn{2}{|c|}{0.0} & \\
\hline $5+$ cups $/ d$ & \multicolumn{2}{|c|}{0.0} & \multicolumn{2}{|c|}{$0 \cdot 4$} & \\
\hline Regular use of table salt after cooking (\% yes) & \multicolumn{2}{|c|}{$42 \cdot 7$} & \multicolumn{2}{|c|}{$29 \cdot 6$} & 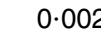 \\
\hline Consumption of meat products highly preserved in salt (times/week) & $1 \cdot 2$ & $2 \cdot 5$ & $1 \cdot 1$ & $1 \cdot 8$ & $0 \cdot 47$ \\
\hline Fast food consumption (times/week) & $1 \cdot 6$ & $1 \cdot 0$ & $1 \cdot 6$ & 0.9 & 0.39 \\
\hline Intake of vitamin or other dietary supplements (\% yes) & \multicolumn{2}{|c|}{$10 \cdot 6$} & \multicolumn{2}{|c|}{$14 \cdot 5$} & $0 \cdot 19$ \\
\hline Regular use of non-stick cookware (\% yes) & \multicolumn{2}{|c|}{$46 \cdot 3$} & \multicolumn{2}{|c|}{$40 \cdot 8$} & $0 \cdot 3$ \\
\hline
\end{tabular}

Data are presented as means and standard deviations or frequencies.

The $t$ test or Mann-Whitney ranked test was applied to evaluate differences in the distributions of the tested variables between cases and controls.

Table 2 Results from multiple logistic regression analyses that evaluated the association of certain nutritional behaviours in the context of the Mediterranean diet (as assessed using the MedDietScore) with colorectal cancer (CRC) in a sample of CRC cases ( $n 250)$ and controls group-matched for age and sex (n 250), Athens, Greece, December 2009-December 2010

\begin{tabular}{|c|c|c|c|c|}
\hline & \multicolumn{2}{|c|}{ Model 1} & \multicolumn{2}{|c|}{ Model 2} \\
\hline & OR & $95 \% \mathrm{Cl}$ & OR & $95 \% \mathrm{Cl}$ \\
\hline Number of meals daily (per 1 meal) & $0 \cdot 74$ & $0.61,0.89$ & 0.75 & $0.62,0.90$ \\
\hline $\begin{array}{l}\text { Regular use of table salt after cooking (yes } v . \text { no) } \\
\text { Coffee intake }\end{array}$ & $1 \cdot 62$ & $1 \cdot 05,2 \cdot 49$ & $1 \cdot 41$ & $0 \cdot 90,2 \cdot 20$ \\
\hline 1 cup/d $v$. none & $1 \cdot 02$ & $0 \cdot 52,2 \cdot 01$ & $1 \cdot 00$ & $0.50,2 \cdot 00$ \\
\hline $2-3 \mathrm{cups} / \mathrm{d}$ v. none & $1 \cdot 10$ & $0 \cdot 55,2 \cdot 19$ & $1 \cdot 11$ & $0 \cdot 55,2 \cdot 24$ \\
\hline 4-5 cups/d v. none & $3 \cdot 27$ & $1 \cdot 09,9 \cdot 80$ & $2 \cdot 85$ & $0 \cdot 93,8 \cdot 71$ \\
\hline $5+$ cups $/ d v$. none & 0.99 & $0.22,4.53$ & 0.61 & $0 \cdot 13,2 \cdot 90$ \\
\hline Regular use of non-stick cookware (yes $v$. no) & 1.57 & $1 \cdot 03,2 \cdot 40$ & $1 \cdot 50$ & $0 \cdot 97,2 \cdot 31$ \\
\hline MedDietScore (per $1 / 55$ unit) & - & - & 0.89 & $0.84,0.94$ \\
\hline
\end{tabular}

Both models were also adjusted for age, sex, BMI, physical activity status, smoking habits and family history of CRC.

table salt was positively associated with a higher likelihood of CRC (model 1, Table 2). However, when the MedDietScore was added in the model in order to account for overall dietary habits, the effect size of the aforementioned associations was reduced slightly (model 2, Table 2). None of the other dietary behavioural characteristics studied here (i.e. times of fast food consumption per week, consumption of meat products highly preserved in salt like ham, bacon and sausages, tea drinking, intake of vitamin or other dietary supplements) were associated with CRC. Moreover, physical activity status was not associated with CRC (OR $=0 \cdot 90,95 \%$ CI $0.58,1.39, P=0.63)$. At this point it should also be mentioned that the MedDietScore was highly inversely associated with the likelihood of having CRC (OR $=0 \cdot 89$, 95\% CI 0.84, 0.94, $P<0 \cdot 001)$; the corresponding C-statistic was $0 \cdot 760(P<0 \cdot 001)$.

\section{Evaluation of the modified-MedDietScore in relation to colorectal cancer}

According to the aforementioned analysis, the number of meals daily, daily coffee intake, use of non-stick cookware and use of added table salt were significantly associated with CRC; therefore they were included in the diet and nutrition behaviour score, the modified-MedDietScore. The mean value of the modified-MedDietScore was much lower for CRC cases as compared with controls (39.0 (SD 4.5) $v$. $41 \cdot 5$ (sD 4.6), $P<0 \cdot 001$ ).

Each one of the aforementioned variables was consecutively added in the MedDietScore and logistic regression models were estimated, adjusted for age, sex, BMI, physical activity status, smoking habits and family history of CRC (Table 3). As can be seen in Table 3, the first variable added in the initial MedDietScore was the number of meals 
Table 3 Results from logistic regression analyses that evaluated the association of the modified-MedDietScore with colorectal cancer (CRC) in a sample of CRC cases ( $n$ 250) and controls group-matched for age and sex ( $n$ 250), Athens, Greece, December 2009-December 2010

\begin{tabular}{lrr}
\hline & OR & $95 \% \mathrm{Cl}$ \\
\hline MedDietScore (per 1/55 units) & $0 \cdot 88$ & $0 \cdot 83,0.92$ \\
MedDietScore+meals/d (per 1/60 units) & $0 \cdot 87$ & $0 \cdot 82,0.91$ \\
MedDietScore+meals/d+cups of coffee/d (per 1/65 units) & $0 \cdot 86$ & $0 \cdot 82,0.91$ \\
MedDietScore+meals/d+cups of coffee/d+non-stick cookware (per 1/70 units) & 0.87 & $0 \cdot 82,0.91$ \\
MedDietScore+meals/d+cups of coffee/d+non-stick cookware+added table salt (per 1/75 units) & 0.87 & $0.83,0.91$ \\
\hline
\end{tabular}

All models were adjusted for age, sex, BMI, physical activity status, smoking habits and family history of CRC.

consumed daily. Adding this variable, the new modifiedMedDietScore (MedDietScore+number of meals) had a good classification rate (C-statistic $=0 \cdot 769, P<0 \cdot 001)$, while a 1-unit increase in value of the modified-MedDietScore was now associated with 13\% lower likelihood of having CRC. The second variable added in the modified score was the daily intake of cups of coffee. The correct classification rate was improved (C-statistic $=0 \cdot 772, P<0 \cdot 001$ ), with a 1 -unit increase in the modified-MedDietScore being associated with $14 \%$ lower likelihood of having CRC. The next variable added to the modified-MedDietScore was the use of nonstick cookware; the correct classification rate was further improved (C-statistic $=0 \cdot 791, P<0 \cdot 001$ ). The last variable added was the use of table salt and the correct classification rate was further improved slightly (C-statistic $=0.792$, $P<0 \cdot 001)$. The order of the variables added in the basic score was according to their significance in the exploratory analysis. Finally, data analysis showed that a 1-unit increase in the latest version of the modified-MedDietScore was associated with $13 \%$ lower odds $(P<0 \cdot 001)$ of having CRC (Table 3).

\section{Discussion}

In the present work the association between certain nutritional behaviours and CRC was evaluated separately as well as in the context of the Mediterranean diet. The number of meals consumed daily, coffee drinking, added table salt consumption and non-stick cookware use were associated with CRC. Moreover, a diet and nutrition behaviour score, the modified-MedDietScore, was also presented here. Comparing the new modified-MedDietScore with its initial version, it should be mentioned that there was an increase in its discriminating ability, suggesting that the inclusion of the aforementioned behaviours seems to confer a better understanding of the relationship between diet and CRC.

According to the 2007 second expert report of the World Cancer Research Fund/American Institute for Cancer Research, a dietary pattern close to that of the Mediterranean diet (i.e. a dietary pattern emphasizing fruits and vegetables, whole grains, legumes, nuts, seeds and low-fat dairy products) could be recommended for all people at risk of cancer and cancer survivors ${ }^{(10)}$. Comparing the first expert report in 1997 and the latest, it is obvious that the 'protective' or 'detrimental' effect of specific food groups or components was downgraded from 'convincing' to 'probable'. Both considerations and the holistic approach of nutrition developed in the last decades indicate that neither a single nutrient nor a single dietary pattern is able to explain the epidemiology of chronic diseases. It is more evident nowadays that not only what people eat, but also the frequency with which people consume food, how it is preserved and cooked have an effect on their health status. Combining dietary and nutritional behaviour information in relation to CRC is an area of much interest since data are limited and the nutritional epidemiology of this type of cancer is not clear.

It has been reported that having frequent meals is related to better lifestyle and dietary status and better glycaemic control $^{(17)}$, whereas, in contrast, a small number of meals (i.e. less than three daily) has been associated with smoking, higher alcohol consumption, lower leisure-time physical activity ${ }^{(17,31)}$, increased visceral fat and insulin resistance $^{(32)}$. Insulin is considered to increase insulin-like growth factor (IGF)-I. Insulin and IGF-I receptors have been found in both normal and malignant cells of human colonic mucosa, and thus insulin and IGF-I can stimulate their proliferation. Moreover, circulating levels of IGF-I and -II and IGF-binding protein (IGFBP)-III, which modulates access of IGF-I to IGF receptors, were related to subsequent CRC risk ${ }^{(16)}$. The high levels of C-reactive protein, TNF- $\alpha$ and IL- 6 that are observed in the plasma of obese individuals have been associated with the development of insulin resistance, elevated plasma levels of insulin and IGF-I, and low levels of IGFBP. In vitro data showed that exposure of colorectal cancer cells to insulin, IGF-1 and TNF- $\alpha$ led to increased rates of proliferation and impaired apoptosis, both of which favour tumorigenesis ${ }^{(33)}$. Recent reports indicated that leptin, a secretory product of adipocytes, also present in abnormally high levels in the plasma of obese people, promotes mitosis and suppresses apoptosis in colonic epithelial cells ${ }^{(34)}$.

Moderate coffee consumption has been associated with reduced risk for CRC in some case-control studies ${ }^{(13-15)}$, whereas cohort studies showed inconsistent results; animal studies on the effects of coffee constituents, such as caffeine, kahweol and cafestol, on colon tumorigenesis have also shown conflicting results. Based on these data 
we decided to assign the greater value (most protective effect) to moderate coffee consumption of $2-3 \mathrm{cups} / \mathrm{d}^{(35)}$.

Added table salt is well documented to play a role in CVD as well as in metabolic syndrome. Although a lowsodium diet is beneficial for overall health, added salt is not related to cancer, in spite the fact that salted foods are highly connected to gastric cancer. However, these conclusions came from studies in populations with high consumption of this type of foods ${ }^{(20)}$. Our data revealed that Greek people rarely consume salted foods (only $6 \%$ reported a consumption frequency of once per week) and intake of other processed food of animal origin high in salt was not correlated to increased risk, while the major source of salt was table salt. High sodium intake is responsible for hypertension, metabolic syndrome and $\mathrm{CVD}^{(36)}$, and there are some preliminary data that it might be connected to elevated systemic inflammation ${ }^{(37)}$; which all indicates that table salt may have a secondary contribution to CRC epidemiology through the promotion of the aforementioned diseases.

Finally, the frequency of use of non-stick cookware is considered to be related to CRC by two possible pathways. The first one represents the way the foods are prepared. Non-stick cookware is used in hazardous cooking methods (i.e. broiling, frying, grilling or barbecuing) at high temperatures mainly for meat, poultry or fish. All of these procedures are strongly related to the formation of HCA. Moreover, the degree of browning (lightly, moderately, strongly or extremely browned) increases the formation of $\mathrm{HCA}^{(22)}$ and Greek people most of the time consume welldone meat. PAH are also produced when meat is cooked at high temperature as a result of the pyrolysis of fat. Both have been found to interact with the polymorphisms in carcinogen-metabolizing enzymes. Evaluating the genetic variants in fifteen enzymes involved in the metabolism of carcinogens in overcooked meat (cytochrome P540, glutathione-S-transferase, UGT-glucuronosyltransferases, SULT, NAT, mEH, AHR), CYP2C9 and NAT2 variants were associated with CRC risk. Red meat intake was associated with increased CRC risk regardless of genotype, but CYP1B1 combined variant and SULT1A1-638G $>$ A variant significantly modified the association between red meat doneness intake and $\mathrm{CRC}^{(38)}$. The second possible pathway implicates the composition of this type of cookware. The coating is usually Teflon, which contains PFOA and PFOS, both suspected carcinogens, and the possible mechanism is the generation of oxidative stress ${ }^{(21)}$. Although they are considered to be environmental risk factors and the main source for human consumption is through water contamination, a recent Greek study revealed no differences in the blood levels of PFOA and PFOS between three groups: one semi-urban (from Athens), one rural and one of cancer patients, and showed that these concentrations are in line with those in other European countries ${ }^{(39)}$. These data allow us to consider non-stick cookware as another major available source. Data from animal studies support that both of these chemicals can cause several types of tumour and may have many toxic effects on the immune system, liver and endocrine system. Moreover, PFOA has been found to be associated with increased blood cholesterol, but all of the above data are sparse in human subjects ${ }^{(40)}$.

One of the strengths of the present study is that it proposes, for the first time, a dietary score containing variables not only representative of a dietary pattern, but also dietary behaviours related to everyday practice. More and more research is being done in the field of how foods are preserved and which cooking methods are used. It is more than obvious that public health promotion is strongly related not only strictly to dietary guidelines, but also means the provision of appropriate food managing practices. Our innovation is in accordance with the latest guidelines on food and nutrition for the prevention of cancer ${ }^{(10)}$. The advantages of using diet scores to allocate the high-risk individuals are also of considerable importance for public health. The present work could be described as the combination of theoretical knowledge on nutrition aspects and how they correlate with cancer prevalence, as well as information on dietary behaviours and how they might influence the role of nutrition and food in cancer epidemiology. Based on the globally increasing numbers of new cancer cases (with CRC taking the third place in Western countries) and cancer survivors, it is of great importance to propose a reliable, validated and easy-to-use dietary score in order to discriminate people at high risk for the development or recurrence of cancer (CRC in our case). Moreover, the proposed score incorporates all current knowledge about the development of such indices, i.e. the large scale of the components scoring system, the lack of a high level of intra-correlation between them and the good level of inter-correlation with the studied outcome. The major limitation of the present study is the recall bias, as in all case-control studies. However, an effort was made to minimize this limitation by choosing newly diagnosed patients and enrolling all necessary participants within a small period time, approximately 12 months. Moreover, the people who collected the data were trained, limiting the bias between investigators. It should also be mentioned that, like in all case-control studies, our findings cannot support a causative association between dietary factors and CRC, but can set the hypotheses for further investigations, mainly cohort studies.

\section{Conclusions}

Certain nutritional behaviours seem to confer a better understanding of the relationship between diet and CRC. Moreover, the inclusion of these behaviours in the context of a healthy dietary evaluation instrument, the modified-MedDietScore, showed it to be a promising prescreening tool, able to better allocate people at high risk 
for developing CRC. Thus, it could be used by health practitioners in primary and secondary prevention as a cost-effective and feasible approach.

\section{Acknowledgements}

This research received no specific grant from any funding agency in the public, commercial or not-for-profit sectors. No conflict of interest exists. N.K. wrote the paper, took part in the acquisition, analysis and interpretation of data and performed the statistical analysis. T.P. critically reviewed the paper. N.S. helped in data acquisition. E.P., A.L. and D.X. critically reviewed the paper. D.B.P. formulated the study concept and design and supervised the study and statistical analysis. Emmanouel Paraskevas critically reviewed the paper. The authors would like to thank all participants of the study, without their support this work would never been done. The authors would also like to thank the field investigators of the study: Efstathia Koloverou, Maria Nikolaou, Eleni Triantafillou, Georgia Georgiou and Vladleva Khudokonenko, as well as the nurses and doctors of the two hospitals.

\section{References}

1. International Agency for Research on Cancer, World Health Organization (2008) GLOBOCAN Cancer Fact Sheets: Colorectal Cancer. http://globocan.iarc.fr/factsheet.asp (accessed April 2012).

2. Panagiotakos DB (2008) $\alpha$-Priori vs. $\alpha$-posterior methods in dietary pattern analysis: a review in nutrition epidemiology. Nutr Bull 33, 311-315.

3. Waijers PMCM, Feskens EJM \& Ocké MC (2007) A critical review of predefined diet quality scores. Br J Nutr 97, 219-231.

4. Cottet V, Touvier M, Fournier A et al. (2009) Postmenopausal breast cancer risk and dietary patterns in the E3N-EPIC prospective cohort study. Am J Epidemiol 170, 1257-1267.

5. Fung TT, Hu FB, McCullough ML et al (2006) Diet quality is associated with the risk of estrogen receptor-negative breast cancer in postmenopausal women. J Nutr 136, 466-472.

6. Jansen MC, Bueno-de-Mesquita HB, Buzina R et al. (1999) Dietary fiber and plant foods in relation to colorectal cancer mortality: the Seven Countries Study. Int J Cancer 81, 174-179.

7. Cottet V, Bonithon-Kopp C, Kronborg O et al. (2005) Dietary patterns and the risk of colorectal adenoma recurrence in a European intervention trial. Eur J Cancer Prev 14, 21-29.

8. Reedy J, Mitrou PN, Krebs-Smith SM et al. (2008) Indexbased dietary patterns and risk of colorectal cancer, the NIH-AARP Diet and Health study. Am J Epidemiol 168, 38-48.

9. Bosetti C, Gallus S, Trichopoulou A et al. (2003) Influence of the Mediterranean diet on the risk of cancers of the upper aerodigestive tract. Cancer Epidemiol Biomarkers Prev 12, 1091-1094.

10. World Cancer Research Fund \& American Institute for Cancer Research (2007) Food, Nutrition, Physical Activity and the Prevention of Cancer: A Global Perspective. Washington, DC: AICR.
11. Peters U, Chatterjee N, McGlynn KA et al. (2004) Calcium intake and colorectal adenoma in a US colorectal cancer early detection program. Am J Clin Nutr 80, 1358-1365.

12. Oh K, Willett WC, Wu K et al. (2007) Calcium and vitamin $\mathrm{D}$ intakes in relation to distal colorectal adenoma in women. Am J Epidemiol 165, 1178-1186.

13. Sugiyama K, Kuriyama S, Akhter M et al. (2010) Coffee consumption and mortality due to all causes, cardiovascular disease, and cancer in Japanese women. $J$ Nutr 140, 1007-1013.

14. Woolcott CG, King WD \& Marrett LD (2002) Coffee and tea consumption and cancers of the bladder, colon and rectum. Eur J Cancer Prev 11, 137-145.

15. Binns CW, Lee AH \& Fraser ML (2008) Tea or coffee? A case study on evidence for dietary advice. Public Health Nutr 11, 1132-1141.

16. Franceschi S, Dal Maso L, Augustin L et al. (2001) Dietary glycemic load and colorectal cancer risk. Ann Oncol 12, 173-178.

17. Sierra-Johnson J, Urdén AL, Linestrand M et al. (2008) Eating meals irregularly: a novel environmental risk factor for the metabolic syndrome. Obesity (Silver Spring) 16, 1302-1307.

18. Schnohr P, Grønbæk M, Petersen L et al. (2005) Physical activity in leisure-time and risk of cancer: 14-year follow-up of 28,000 Danish men and women. Scand J Public Health 33, 244-249.

19. Hill MJ (1999) Diet, physical activity and cancer. Public Health Nutr 2, 397-401.

20. Takachi R, Inoue M, Shimazu T et al. (2010) Consumption of sodium and salted foods in relation to cancer and cardiovascular disease: the Japan Public Center-based Prospective Study. Am J Clin Nutr 91, 456-464.

21. Eriksen KT, Raaschou-Nielsen O, Sørensen M et al. (2010) Genetoxic potential of the perfluorinated chemicals PFOA, PFOS, PFBS, PFNA and PFHxA in human HepG2 cells. Mutat Res 700, 39-43.

22. Rohrmann S, Hermann S \& Linseisen J (2009) Heterocyclic aromatic amine intake increases colorectal adenoma risk: findings from a prospective European cohort study. Am J Clin Nutr 89, 1418-1424.

23. Vogelaar I, van Ballegooijen M, Schrag D et al. (2006) How much can current interventions reduce colorectal cancer mortality in the US? Cancer 107, 1624-1633.

24. Shankaran V, Luu TH, Nonzee N et al. (2009) Costs and cost effectiveness of a health care provider-directed intervention to promote colorectal cancer screening. J Clin Oncol 27, 5370-5375.

25. Bountziouka V, Bathrellou E, Giotopoulou A et al. (2011) Development, repeatability and validity regarding energy and macronutrient intake of a semi-quantitative food frequency questionnaire: methodological considerations. Nutr Metab Cardiovasc Dis (Epublication ahead of print version).

26. Panagiotakos DB, Pitsavos C, Arvaniti F et al. (2007) Adherence to the Mediterranean food pattern predicts the prevalence of hypertension, hypercholesterolemia, diabetes and obesity, among healthy adults; the accuracy of the MedDietScore. Prev Med 44, 335-340.

27. Kourlaba G \& Panagiotakos D (2009) The number of index components affects the diagnostic accuracy of a diet quality index: the role of intracorrelation and intercorrelation structure of the components. Ann Epidemiol 19, 692-700.

28. Kontou N, Panagiotakos DB, Psaltopoulou T et al. (2011) Discriminating ability of the MedDietScore in relation to gastrointestinal tract cancer: a sensitivity-specificity analysis. Agro Food Ind Hi Tech 22, 42-45.

29. Finucane MM, Stevens GA, Cowan MJ et al. (2011) National, regional, and global trends in body-mass index since 1980: systematic analysis of health examination 
surveys and epidemiological studies with 960 countryyears and 9.1 million participants. Lancet 337, 557-567.

30. Grundy SM, Brewer B, Cleeman JI et al. (2004) Definition of metabolic syndrome. Circulation 109, 433-438.

31. Holmbäck I, Ericson U, Gullberg B et al. (2010) A high eating frequency is associated with an overall healthy lifestyle in middle-aged men and women and reduced likelihood of general and central obesity in men. BrJ Nutr 104, 1065-1073

32. Kershaw EE \& Flier JS (2004) Adipose tissue as an endocrine organ. J Clin Endocrinol Metab 89, 2548-2556.

33. Johnson IT \& Lund EK (2007) Review article: nutrition, obesity and colorectal cancer. Aliment Pharmacol Ther 26, 161-181.

34. Hoda MR, Keely SJ, Bertelsen LS et al. (2007) Leptin acts as a mitogenic and antiapoptotic factor for colonic cancer cells. Br J Surg 94, 346-354.

35. Arab L (2010) Epidemiologic evidence on coffee and cancer. Nutr Cancer 62, 271-283.
36. Brown IJ, Tzoulaki I, Candeias V et al. (2009) Salt intakes around the world: implications for public health. Int $J$ Epidemiol 38, 791-813.

37. Fogarty AW, Lewis SA, McKeever TM et al. (2009) Is higher sodium intake associated with elevated systemic inflammation? A population-based study. Am J Clin Nutr 89, 1901-1904.

38. Cotterchio M, Boucher BA, Manno M et al. (2008) Red meat intake, doneness, polymorphisms in genes that encode carcinogen-metabolizing enzymes and colorectal cancer risk. Cancer Epidemiol Biomarkers Prev 17, 3098-3107.

39. Vassiliadou I, Costopoulou D, Ferderigou A et al. (2010) Levels of perfluorooctanesulfonate (PFOS) and perfluorooctanoate (PFOA) in blood samples from different groups of adults living in Greece. Chemosphere 80, 1199-1206.

40. Steenland K, Fletcher T \& Savitz DA (2010) Epidemiologic evidence on the health effects of perfluorooctanoic acid (PFOA). Environ Health Perspect 118, 1100-1108. 